\title{
Impacto da Infecção Chagásica sobre Algumas Características Demográficas: Resultados de um Estudo Ecológico
}

\author{
Impact of Chagas' Infection on Some Demographic Characteristics: \\ Results of an Ecological Study
}

\author{
Maurício L. Barreto ${ }^{1}$ \\ Maria Eliane B. Andrade ${ }^{1}$
}

\section{BARRETO, M. L. E ANDRADE, M. E. B. Impact of Chagas'Infection on Some Demographic Characteristics: Results of an Ecological Study. Cad. Saúde Públ., Rio de faneiro, 10 (supplement 2): 273-280, 1994.}

This study aimed to analyze the impact of $\underline{T}$. cruzi infection on some demographic characteristics in the State of Bahia. It is a place where Chagas' disease is endemic in general. In the endemic areas, similar infection rates were observed in males and females. However, males, mainly those aged 30 or more, had higher mortality rates than females. Analyses were conducted on a set of secondary data from several different sources. They included prevalence of T. cruzi infection, specific mortality related to Chagas' disease, and demographic characteristics. The data were aggregated at two different levels: municipality and homogeneous micro-regions. Significant positive correlations were observed between prevalence of $\underline{T}$. cruzi infection and mortality rates. These indicators, in turn, were correlated with the proportion of widows and widowers in the population. Findings were higher for widows. In areas where prevalence of $\underline{T}$. cruzi infection was $15 \%$ or greater, there were $22 \%$ more widows as compared to infection free areas. Places with high prevalence presented some skew in the age structure, and there was a consistent decrease in the proportion of males in the 35-44 age group. $A$ possible confounding effect of migration on these finding was not demonstrated.

Key words: Chagas' Disease; Trypanosoma cruzi Infection; Demographic Structure; Ecological Study

\section{INTRODUÇÃO}

A doença de Chagas, resultante da infecção pelo Trypanossoma cruzi, é endêmica em algumas regióes do Brasil, destacando-se a região Nordeste, da qual o Estado da Bahia faz parte. Nesse estado, a prevalência média é de $5,4 \%$, o quinto índice mais alto entre os demonstrados pelos estados brasileiros, de acordo com o inquérito sorológico nacional realizado no período de 1975 a 1980 (Camargo et al., 1984). Essa média, entretanto, oculta grandes variações locais e regionais. A infecção chagá-

${ }^{1}$ Departamento de Medicina Preventiva da Universidade Federal da Bahia. Rua Padre Feijó 29, $4^{\circ}$ andar, Salvador, BA, 40110-170, Brasil. sica está associada a uma série de efeitos mórbidos sobre o organismo humano, destacando se alterações cardiológicas, que se tornam, assim, importante causa de morbidade e mortalidade nas áreas com alta prevalência.

A doença de Chagas, enquanto possa ser causa de óbito em qualquer grupo etário, é descrita, em estudos epidemiológicos efetuados em populações com altas prevalências de infecção, como associada à uma maior taxa de mortalidade no grupo populacional de maiores de 30 anos de idade, do sexo masculino (Puigbó \& Garcia-Barrios, 1966; Dias \& Kloetzel, 1968; Kloetzel \& Dias, 1968; Maguirre et al., 1987; Mota et al., 1990). Até o momento, o impacto dessas altas e seletivas taxas de mortalidade sobre as características demográficas das populações afetadas nunca foi devidamente 
investigado. Assim, este estudo tem como objetivo analisar o impacto dessa infecção sobre algumas características demográficas do Estado da Bahia, onde a endemia ocorre de forma desigual em seu espaço geográfico, incluindo áreas com altas prevalências.

\section{METODOLOGIA}

Em 1980, o Estado da Bahia era composto por 336 municípios, com área total de $561.026 \mathrm{~km}^{2}$ e população de aproximadamente 10.000.000 habitantes, conforme resultados do censo demográfico realizado naquele ano. A utilização dessa base de municípios, bem como dos dados do referido censo, foi devida à proximidade com o ano de 1979, período da conclusão do inquérito sorológico no estado.

As variáveis utilizadas neste estudo foram originadas de três fontes distintas: a) a prevalência da infecção chagásica (PIC) foi obtida do inquérito sorológico patrocinado pela Superintendência de Campanhas de Saúde Pública (Sucam), no período de 1975 a 1980; esse inquérito, realizado em nível nacional, constou de uma amostra estratificada em vários estágios, que permitiu estimar as prevalências no nível dos municípios (Camargo et al., 1984); b) os dados demográficos - estrutura etária: percentual de indivíduos em cada faixa etária com relação à população total, por sexo; percentual de viúvos(as) na faixa etária de 15 anos e mais, com relação a população total nessa mesma faixa; e percentual de imigrantes na população geral - foram provenientes do censo demográfico realizado no ano de 1980 (IBGE, 1980a, 1980b); c) as taxas de mortalidade por doença de Chagas (TMDC) e de mortalidade proporcional por doença de Chagas (MPDC) foram extraídas do Sistema de Informação de Mortalidade do Ministério da Saúde.

Para as análises, os dados foram agregados em dois níveis: municípios e microrregiões homogêneas (MRH). Os municípios constituemse nas menores subdivisões com autonomia administrativa de cada estado da Federação. O Estado da Bahia, em 1980, era composto de 336 municípios, dos quais sete foram excluídos do estudo, por não ter prevalências determinadas pelo inquérito sorológico. As MRHs eram em número de 26 e são constituídas pela agre- gação de vários municípios, a partir de características que as homogenizam com relação a aspectos geográficos, sociais e econômicos.

Os bancos de dados, com todas as variáveis utilizadas, foram organizados em microcomputador, e as análises de correlação e regressão, realizadas segundo o pacote SPSS-PC+.

\section{RESULTADOS}

A prevalência média da infecção chagásica no Estado da Bahia era de 5,4\%, variando de zero a $63 \%$ no nível de municípios (Figura 1) e de $0,12 \%$ a $16,4 \%$ no nível das MRHs. A taxa de mortalidade por doença de Chagas (TMDC) assumiu no estado o valor de $3,5 / 10^{5} \mathrm{hab}$. e variou de zero a $67,9 / 10^{5} \mathrm{hab}$., entre os municípios, e de zero a $15,4 / 10^{5} \mathrm{hab}$., entre as MRHs, enquanto a mortalidade proporcional por doença de Chagas foi de 1,4\% para a média do estado, variando de zero a $12,5 \%$, entre os municípios, e de zero a $1,9 \%$, entre as MRHs. Os coeficientes de correlação ( $r$ ) entre as PICs e as TMDC e MPDC mostraram correlações positivas e estatisticamente significantes, tanto no nível de município como a nível de MRH (Tabela 1).

TABELA 1. Coeficientes de Correlação (r) entre as Taxas de Prevalência da Infecção pelo T. cruzi com as Taxas de Mortalidade (TMDC) e as Mortalidades Proporcionais por Doença de Chagas (MPDC), no Nível de Municípios e Microrregiões Homogêneas (MRH) do Estado da Bahia - 1980

\begin{tabular}{lcccc}
\hline \hline & \multicolumn{2}{c}{ Municípios } & \multicolumn{2}{c}{ MRH } \\
\cline { 2 - 5 } & $\mathrm{r}$ & $\mathrm{p}$ & $\mathrm{r}$ & $\mathrm{p}$ \\
\hline TDMC & .27 & .000 & .41 & .019 \\
& & & & \\
MPDC & .36 & .000 & .68 & .000 \\
\hline \hline
\end{tabular}

As correlações entre as prevalências e a proporção de viúvos(as) na população estão apresentadas na Tabela 2 . No nível de município, esses valores foram de $0,10(\mathrm{p}=0,034) \mathrm{e}$ $0,22(\mathrm{p}=0,000)$ para viuvez masculina $\mathrm{e}$ feminina, respectivamente. No nível das MRHs, as correlações assumem valores mais altos, 
FIGURA 1. Prevalência da Doença de Chagas no Estado da Bahia - 1979

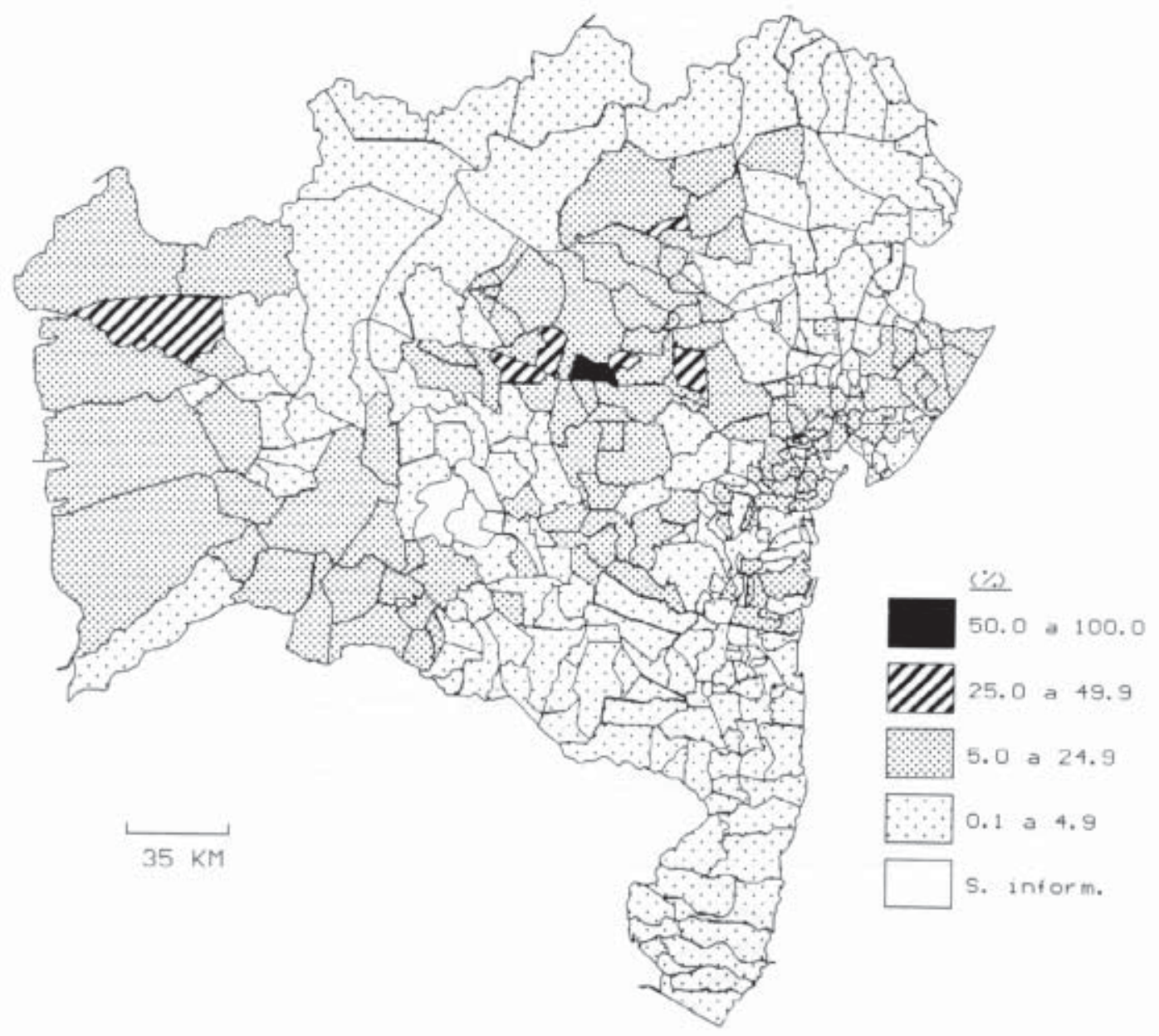

Fonte: Inquérito Sorológico Nacional, 1979. 
destacando se, entretanto, a viuvez feminina ( $\mathrm{r}$ $=0,38 ; \mathrm{p}=.0027)$, pois, para o sexo masculino, a correlação não foi significativa $(r=0,22 ; p=$ $0,13)$. Quando os mesmos indicadores de viuvez são correlacionados com TMDC e MPDC, os resultados são sempre positivos e significativos, assumindo, porém, valores mais altos no sexo feminino (Tabela 3). Quando os municípios foram agregados em quatro faixas $(0 ; 0,1-4,9 \%$; $5,014.9 \% ; 15,0+\%)$ a proporção de viúvas variou de $4,0 \%, 4,1 \%, 4,7 \%$ e $4,9 \%$, respectivamente, o que significa excesso de viúvas de $22,5 \%$ nos municípios com a PIC de $15 \%$ ou mais, quando comparados com aqueles indenes para a infecção chagásica (Tabela 4).
TABELA 2. Coeficientes de Correlação (r) entre as Taxas de Infecção pelo T. cruzi com as Proporções de Viúvos (PVM), Viúvas (PVF) e Viúvos + Viúvas (PVT), no Nível de Municípios e Microrregiões Homogêneas $(\mathrm{MRH})$ do Estado da Bahia - 1980

\begin{tabular}{lcccc}
\hline \hline & \multicolumn{2}{c}{ Municípios } & \multicolumn{2}{c}{ MRH } \\
\cline { 2 - 5 } & $\mathrm{r}$ & $\mathrm{p}$ & $\mathrm{r}$ & $\mathrm{p}$ \\
\hline PVM & .10 & .034 & .22 & .235 \\
PVF & .22 & .000 & .38 & .027 \\
PVT & .220 & .000 & .36 & .034 \\
\hline \hline
\end{tabular}

TABELA 3. Coeficientes de Correlação (r) entre as Taxas de Mortalidade (TMDC) e Mortalidades Proporcionais por Doença de Chagas (MPDC) com as Proporções de Viúvos (PVM) e Viúvas (PVF) nos Municípios e Microrregiões Homogêneas do Estado da Bahia - 1980

\begin{tabular}{|c|c|c|c|c|c|c|c|c|}
\hline & \multicolumn{4}{|c|}{ Municípios } & \multicolumn{4}{|c|}{ MRH } \\
\hline & \multicolumn{2}{|c|}{ PVM } & \multicolumn{2}{|c|}{ PVF } & \multicolumn{2}{|c|}{ PVM } & \multicolumn{2}{|c|}{ PVF } \\
\hline & $\mathrm{r}$ & $\mathrm{p}$ & $\mathrm{r}$ & $\mathrm{p}$ & $\mathrm{r}$ & $\mathrm{p}$ & $\mathrm{r}$ & $\mathrm{p}$ \\
\hline TDMC & 0,18 & 0,000 & 0,35 & 0,000 & 0,48 & 0,006 & 0,72 & 0,000 \\
\hline MPDC & 0,09 & 0.049 & 0,12 & 0,016 & 0,29 & 0,006 & 0,54 & 0,002 \\
\hline
\end{tabular}

TABELA 4. Número (NV) e Proporção de Viúvas (PVF) e Razões de Prevalências (RP) por Nível de Prevalência da Infecção pelo T. cruzi dos Municípios do Estado da Bahia - 1980

\begin{tabular}{lccc}
\hline \hline $\begin{array}{l}\text { Nível } \\
\text { de Prevalência (\%) }\end{array}$ & NV & $\begin{array}{c}\text { PVF } \\
(\%)\end{array}$ & $\begin{array}{c}\text { RP } \\
(95 \% \text { LC })\end{array}$ \\
\hline 0 & 9369 & 4,0 & --- \\
$0,1-4,9$ & 86245 & 4,1 & $1,02(1,00-1,05)$ \\
$5,0-14,9$ & 99192 & 4,7 & $1,17(1,15-1,20)$ \\
$15,0-+$ & 15096 & 4,9 & $1,22(1,19-1,26)$ \\
\hline \hline
\end{tabular}

Quando a PIC foi correlacionada com a proporção de indivíduos em cada faixa etária e sexo, observaram se correlações significativas a partir da faixa etária de 35-44 anos. Nesta faixa (35-44 anos) observa se, para o sexo masculino, correlações negativas nos dois níveis de agregação utlizados: $r=-0,16 ; p=0,002$, para município e $r=-0,39 ; p=0,023$, para $M R H$.
Com relação ao sexo feminino, só foi observada correlação significativa no nível municipal $(\mathrm{r}=$ $-0,14 ; \mathrm{p}=0,004) . \mathrm{Na}$ faixa etária seguinte (45-54 anos de idade), as correlações foram negativas para ambos os sexos, sendo, no entanto, significativas apenas para o sexo feminino no nível municipal $(\mathrm{r}=0,15 ; \mathrm{p}=$ $0,004)$. No grupo etário mais idoso (55 anos e 
mais), todas as correlações são positivas, apresentando se significativas para o sexo feminino $(\mathrm{r}=0,14 ; \mathrm{p}=0,006$ e $\mathrm{r}=0,35 ; \mathrm{p}=0,039$, no nível de municípios e MRHs, respectivamente). (Tabela 5)

TABELA 5. Coeficientes de correlação ( $r$ ) entre as Taxas de Infecção pelo T. cruzi com as Proporções da População por Faixa Etária e Sexo no Nível de Municípios e Microrregiões Homogêneas (MRH) do Estado da Bahia - 1980

\begin{tabular}{cccr}
\hline \hline & & \multicolumn{2}{c}{ Nível de Agregação } \\
\cline { 3 - 4 } Idade & Sexo & Municípios & MRH \\
& M & .04 & -.09
\end{tabular}

$0-14$

\begin{tabular}{ccc}
$\mathrm{F}$ & -.05 & -.16 \\
\hline $\mathrm{M}$ & -.01 & -.07
\end{tabular}

\begin{tabular}{rrrr}
$15-24$ & & & \\
& $\mathrm{~F}$ & -.09 & -.23 \\
\hline $\mathrm{M}$ & .02 & .02
\end{tabular}

$25-34$

\begin{tabular}{rrr}
$\mathrm{F}$ & -.08 & -.17 \\
\hline $\mathrm{M}$ & $*_{-} .16$ & $*_{-} .39$
\end{tabular}

$35-44$

\begin{tabular}{rrr}
$\mathrm{F}$ & $*_{-} .14$ & -.16 \\
\hline $\mathrm{M}$ & -.07 & -.28
\end{tabular}

$45-54$

\begin{tabular}{rrr}
$\mathrm{F}$ & $* * .15$ & .27 \\
\hline $\mathrm{M}$ & .02 & .13
\end{tabular}

$55-+$

$\mathrm{F} \quad * * .14 \quad * .35$

$\star{ }^{\star} \mathrm{p}<.05 \quad \star \star \mathrm{p}<.01$

Com o objetivo de explorar possíveis efeitos dos movimentos migratórios nos indicadores demográficos estudados, estes últimos foram correlacionados com a taxa de imigração, TI (proporção de imigrantes com relação à população total), já que não há disponibilidade de taxas emigratórias nos dados censitários. A TI, em relação com o percentual de viúvos/viúvas, a PIC, a TMDC e a PMDC, não mostrou correlações significativas. Com relação aos grupos etários, algumas correlações significativas foram observadas, destacando-se uma, positiva no nível de municípios, no grupo de
45-54 anos de idade, do sexo masculino ( $\mathrm{r}=$ $0,19 ; \mathrm{p}=0,000)$, e correlações negativas, no nível das MRHs, no sexo feminino, nos grupos de $45-54$ e 55 anos e mais $(r=-0,43 ; \mathrm{p}=0,013$ e $\mathrm{r}=-0,41 ; \mathrm{p}=0,020$, respectivamente).

(Tabela 6)

TABELA 6. Coeficientes de Correlação (r) entre as Proporções de Imigrantes com as Proproções da População por Faixa Etária e Sexo no Nível de Municípios e Microrregiões Homogêneas (MRH) do Estado da Bahia - 1980

\begin{tabular}{lccr}
\hline \hline & & \multicolumn{2}{c}{ Nível de Agregação } \\
\cline { 3 - 4 } Idade & Sexo & & \\
\hline & $\mathrm{M}$ & $*_{-.10}$ & .23 \\
$0-14$ & & & \\
& $\mathrm{~F}$ & -.07 & $* * .49$ \\
\hline & $\mathrm{M}$ & .09 & -.10 \\
$15-24$ & & & \\
& $\mathrm{~F}$ & .04 & -.07 \\
\hline & $\mathrm{M}$ & .01 & -.08 \\
$25-34$ & & & \\
& $\mathrm{~F}$ & -.08 & .01 \\
\hline & $\mathrm{M}$ & .00 & .22 \\
$35-44$ & & & \\
& $\mathrm{~F}$ & .07 & -.13 \\
\hline & $\mathrm{M}$ & $* * * .19$ & .11 \\
$45-54$ & & & \\
& $\mathrm{~F}$ & .01 & $*_{-.43}$ \\
\hline & $\mathrm{M}$ & -.01 & -.26 \\
$55-+$ & & & \\
\hline \hline & $\mathrm{F}$ & -.09 & $*_{-.41}$ \\
\hline
\end{tabular}

${ }^{\star} \mathrm{p}<.05 \quad \star \star \mathrm{p}<.01 \quad \star \star \star \mathrm{p}<.001$

\section{DISCUSSÃO}

A infecção chagásica constitui se em grave problema de saúde pública em algumas áreas do Brasil e em outros países do continente americano (Barreto, 1979; Kirchhoff, 1993). Caracteriza-se por ser uma infecção crônica e por estar associada à patologias graves, principalmente do aparelho circulatório. Em estudos de prevalência, em áreas endêmicas, os infectados apresentam 10-20\% de excesso de alterações 
cardíacas (Maguirre et al., 1983; Schenone et al., 1983; Baruffa et al., 1983). Em estudos epidemiológicos longitudinais, os indivíduos infectados apresentam taxas elevadas de problemas cardíacos e mortalidade (Mota et al., 1990; Maguirre et al., 1987; Pereira et al., 1985; Maguirre et al., 1982; Pugliese et al., 1976; Kloetzel \& Dias, 1968). Está bem-documentado em alguns desses estudos, o fato de o maior risco de óbitos de indivíduos infectados ocorrer entre 30 e 50 anos de idade, predominando no sexo masculino, sem, no entanto, deixar de ter importância no sexo feminino.

Tendo em vista que as altas prevalências da infecção se associam com altas taxas de mortalidade, o que neste estudo é traduzido pelas altas correlações entre a PIC e os indicadores de mortalidade (TMDC e MPDC), seria de esperar que uma infecção com características crônicas e de alta letalidade interferisse na estrutura demográfica de populações em que ela ocorre em altas prevalências. Essa hipótese foi demonstrada neste estudo, conforme os resultados apresentados nas Tabelas 2, 4 e 5 . O registro de correlações positivas e de alta significância estatística entre a proporção de viúvas/viúvos na população e a PIC, as quais foram de maior magnitude com relação à viuvez feminina, é o achado mais consistente do estudo. $\mathrm{O}$ fato de esse achado ter sido observado nos dois níveis em que os dados foram agregados - municípios e MRHs torna os resultados mais consistentes. Esse efeito é de grande magnitude, pois traduz-se no fato de que o conjunto dos municípios com prevalência igual ou superior a $15 \%$ apresentou excesso de $22,5 \%$ na freqüência de viúvas, comparada àquela do conjunto de municípios em que a infecção chagásica não foi encontrada. A possibilidade de efeito migratório foi descartada por falta de argumentos lógicos para tal, bem como pela falta de evidências empíricas, na medida em que não houve nenhuma correlação entre percentual de imigrantes e percentual de viúvos/viúvas.

Com relação à idade, os achados, apesar de terem menor grau de consistência do que aqueles referentes à viuvez, foram coerentes com os resultados dos estudos prospectivos, que relatam, entre os infectados, maior mortalidade entre os homens na faixa de 30 a 50 anos de idade. Assim, o achado de correlações negativas e significativas entre a proporção de homens na faixa etária de 35 a 44 anos e a PIC, nos dois níveis de agregação dos dados, é coerente com os achados epidemiológicos. Para o sexo feminino, nessa mesma faixa etária, a correlação negativa foi significativa apenas no nível de municípios, mostrando associação possível, embora menos consistente. Ainda que a composição etária possa sofrer forte influência dos movimentos migratórios, a imigração não exerceu influência marcante na composição da estrutura etária dos municípios e MRHs do Estado da Bahia, já que as correlações entre a proporção de imigrantes e a proporção da população por faixa etária e sexo que apresentaram significância estatística foram pouco consistentes (Tabela 6).

Este estudo, como outros que se utilizam de dados agregados, apresenta problemas conceituais e metodológicos peculiares e sempre controversos, que necessitam de contextualização, para melhor definir a consistência e validade dos seus resultados e sua importância diante dos resultados encontrados em estudos epidemiológicos do tipo transversal e, principalmente, longitudinal, realizados em áreas com alta prevalência da infecção chagásica. Para verificar a validade interna e aumentar a confiabilidade dos resultados encontrados, validouse a PIC, correlacionando-a com os dois indicadores de mortalidade disponíveis - TMDC e MPDC (Tabela 1). Os resultados mostram que os dois grupos de indicadores, provenientes de fontes diversas e totalmente independentes, apresentam alto grau de correlação, demonstrando, de um lado, a representatividade do inquérito sorológico (Camargo et al., 1984) e, do outro, que, apesar das insuficiências do Sistema de Informação de Mortalidade (Andrade et al., 1993), ele oferece informações confiáveis sobre a magnitude e as tendências da mortalidade pela doença de Chagas. Procurou-se também ampliar a consistência dos resultados pela utilização de dois níveis de agregação, valorizando-se aqueles resultados que foram significativos nos dois níveis (Piantadosi et al., 1988; Cleek, 1979). Uma questão pouco clara em tais estudos tem sido a da extensão dos agregados utilizados. Enquanto alguns defendem o uso de áreas menores e, conseqüentemente, mais homogê- 
neas (Morgenstern, 1982), tem sido mostrado que isso gera fenônemos auto-correlativos que afetam a magnitude dos testes de regressão e correlação utilizados (Lazar, 1981). Outro ponto que merece discussão é o da sempre propalada "falácia ecológica" (Selvin, 1958; Robinson, 1950). Esse termo tem gerado diversas confusões e pode ser resumido na idéia de que inferências sobre o agregado não podem ser extrapoladas imediatamente para o indivíduo, questão que não se coloca em estudos como este, em que o objeto central está em torno de características demográficas, portanto, eminentemente coletivas (Barreto \& Alves, 1994).

\section{RESUMO}

BARRETO, M. L. \& ANDRADE, M. E. B. Impacto da Infecção Chagásica sobre Algumas Características Demográficas: Resultados de um Estudo Ecológico. Cad. Saúde Públ., Rio de Janeiro, 10 (suplemento 2): 273-280, 1994.

Este estudo teve por objetivo analisar o impacto da infecção pelo T. cruzi sobre algumas características demográficas do Estado da Bahia, local em que a doença de Chagas se apresenta em forma endêmica. Nas áreas endêmicas, em geral, observam-se taxas de infecção similares entre homens e mulheres, entretanto, entre os primeiros, principalmente na faixa etária de 30 anos e mais, têm-se observado maiores taxas de mortalidade. Foram analisados secundários referentes à prevalência da infecção pelo $T$. cruzi, à estrutura etária e a taxas de mortalidade, provenientes de diferentes fontes e agregados em dois níveis, municípios e microrregiões homogêneas. Observaram-se correlações positivas entre a prevalência da infecção chagásica e os indicadores de mortalidade. Esses indicadores, por sua vez, apresentaram correlações significativas com a proporção de viúvos e viúvas na população, com maior magnitude no segundo grupo. Assim, nas áreas com taxas de infecção pelo T. cruzi de $15 \%$ ou mais, observaram-se $22 \%$ mais viúvas entre as mulheres do que nas áreas indenes. Observou-se ainda que, áreas com maior prevalência da infecção apresentaram desvios em sua estrutura etária, destacando-se menor percentual de homem nas faixa de 35-44 anos de idade. A possível influência do processo migratório nesses achados não foi demonstrada.

Palavras-Chave: Doença de Chagas; Trypanosoma cruzi; Estrutura Demográfica; Estudo Ecológico

\section{REFERÊNCIAS BIBLIOGRÁFICAS}

ANDRADE, A. E. O; BARRETO, M. L. \& SOUZA, M. C. M., 1993. Identificação de fatores determinantes de sub-registro de óbitos em Itaparica -Ba/Brasil. Informe Epidemiológico do SUS, 5: 40-58.

BARRETO, M. L. \& ALVES, P. C., 1994. O coletivo versus o individual na epidemiologia: contradição ou síntese? In: Qualidade de Vida: Compromisso Histórico da Epidemiologia (M. F. F. Lima e Costa \& R. P. Souza., orgs.), pp. 129134, Belo Horizonte: Coopmed/Rio de Janeiro: Abrasco.

BARRETO, M. P., 1979. Epidemiologia. In: Trypanossoma cruzi e Doença de Chagas (Z. Bremer \& Z. Andrade), pp. 89-151, Rio de Janeiro: Guanabara Koogan.

BARUFFA, G.; ALCANTARA-FILHO, A. \& AQUINO-NETO, J. O., 1983. Correlação sorológica eletrocardiográfica para a doença de Chagas em populações rurais não selecionadas do Rio Grande do Sul. Revista da Sociedade Brasileira Medicina Tropical, 16: 130-138.

CAMARGO, M. E; SILVA, G. R.; CASTILHO, E. A. \& SILVEIRA, A. C., 1984. Inquérito sorológico de prevalência de infecção chagásica no Brasil - 1975/1980. Revista do Instituto de Medicina Tropical de São Paulo, 26: 192-204.

CLEEK, R. K., 1979. Cancers and environment: the effect of scale. Social Science and Medicine, 13D: 241-247.

DIAS, J. C. P. \& KLOETZEL, K., 1968. The prognostic value of the eletrocardiographic features of chronic Chagas' disease. Revista do Instituto de Medicina Tropical de São Paulo, 10: 158 -162.

IBGE (Fundação Instituto Brasileiro de Geografia e Estatística), 1980a. Censo Demográfico: Bahia, 1983. In: Recenseamento Geral do Brasil, 1980 (IBGE), vol. 1, t. 3, pp. 44-139, Rio de Janeiro: IBGE. 
, 1980b. Censo Demográfico: Bahia, 1983. In: Recenseamento Geral do Brasil, 1980 (IBGE), vol. 1, t. 4, pp. 393-397, Rio de Janeiro: IBGE.

KIRCHHOFF, L. V.,1993. Chagas' disease. American trypanossomiasis. Infectious Disease Clinics of North America, 7: 487-502.

KLOETZEL, K. \& DIAS, J. C. P., 1968. Mortality in Chagas' disease: life-table for the period 1949-1967 in an unselected population. Revista do Instituto de Medicina Tropical de São Paulo, 10: 05-08.

LAZAR, P., 1981. Geographical correlations between disease and environmental exposures. In: Perspectives Medical Statistics (E. F. Bithel \& R. Coppi, eds.), pp. 21-38, London: Academic Press.

MAGUIRRE, J. H.; MOTT, K. E.; LEHMAN, J. S.; HOFF, R.; MUNIZ, T. M.; GUIMARÃES, A. C.; SHERLOCK, I. \& MORROW, R. H., 1983. Relationship of eletrocardiographic abnormalities and seropositivity to Trypanosoma cruzi with in a rural community in Northeast Brazil. American Heart Fournal, 105: 287-294.

MAGUIRRE, J. H.; HOFF, R.; SHERLOCK, I.; GUIMARÃES, A. C.; SLEICH, A..C.; RAMOS, N. B.; MOTT, K..E. \& WELLER, T..H., 1987. Cardiac morbitity and mortality due to Chagas' disease: prospective eletrocardiographic study of a Brazilian community. Circulation, 75: 11401145.

MAGUIRRE, J. H.; MOTT, K..E.; SOUZA, J. A. A.; ALMEIDA, E. C.; RAMOS, N. B. \& GUIMARÃES, A. C., 1982. Eletrocardiographic classification and abbreviated lead system for population-based studies of Chagas' disease. Bulletin of the Pan American Health Organization, 16: 47-58.

MORGENSTERN, H., 1982. Uses of ecologic analysis in epidemiological research. American Fournal of Public Health, 72: 1336-1344.
MOTA, E. A.; GUIMARÃES, A. C.; SANTANA, O. O.; SHERLOCK, I.; HOFF, R. \& WELLER, T. H., 1990. A nine year prospective study of Chagas' disease in a defined rural population in Northeast Brazil. American Fournal of Tropical Medicine and Hygiene, 42: 429-440.

PEREIRA, J. B.; WILLCOX, H. P. \& COURA, J. R., 1980. Morbidade da doença de Chagas. III. Estudo longitudinal, de seis anos, em Virgem da Lapa, MG, Brasil. Memórias do Instituto Oswaldo Cruz, 80: 63-71.

PIANTADOSI, S.; BYAR, D. P. \& GREEN, S. B., 1988. The ecological fallacy. American fournal of Epidemiology, 127: 893-904.

PUGLIESE, C.; LESSA, I. \& SANTOS-FILHO, A., 1976. Estudo de sobrevida na miocardite crônica de Chagas descompensada. Revista do Instituto de Medicina Tropical de São Paulo, 18: 191201.

PUIGBÓ, J. J.; RHODE, J. N. \& GARCIA-BARRIOS, H., 1966. Clinical and epidemiological study of chronic heart involvement in Chagas' disease. Bulletin of the World Health Organization, 34: 655-669.

ROBINSON, W. S., 1950. Ecological correlations and the behavior of individuals. American Sociology Review, 15: 351-357.

SELVIN, H. C., 1958. Durkheim's "Suicide" and problems of empirical research. American Fournal of Sociology, 63: 607-619.

SCHENONE, H.; PEREZ-OLEA, J. \& CONTRERAS, M. C., 1983. Enfermedad de Chagas en Chile. Sectores rurales. Frecuencia de alteraciones eletrocardiograficas en 1714 personas con serologia positiva y 8276 personas con serologia negativa. Boletin Chileno de Parasitologia, 38: 67-68. 